\title{
Hybrid Biogeography/Complex-based Optimization
}

\author{
Chen Wang \\ Dept of Mechanical Engineering \\ Hubei University of Automotive Technology \\ Shiyan, China \\ e-mail:wangc_jx@huat.edu.cn
}

\author{
Yang Yang \\ Shanghai Key Laboratory of Intelligent Manufacturing and \\ Robotics \\ Shanghai University \\ Shanghai,China
}

\begin{abstract}
The optimization of complex systems is a very difficult problem in modern engineering technology. It is with multi-subsystems, multi-objectives and multi-constraints. In this paper, a novel solution to the complex systems optimization called HBBO/Complex. HBBO/Complex adapted from biogeography-based optimization (BBO) and combined the simulated annealing (SA). The inferior migrated islands will not be selected unless they pass the Metropolis criterion of SA. This method can prevent the local optimal solution. Compared with typical existing many-objective optimization algorithms, HBBO/complex has better convergence characteristics. The results confirm the $\mathrm{HBBO} / \mathrm{complex}$ provides the best performance in the benchmark problems.
\end{abstract}

\section{Keywords—biogeography; SA; many-objectives optimization}

\section{INTRODUCTION}

Multi-objective evolutionary algorithms (MOEAs) are well-suited for solving numerous multi-objective problems with two or three objectives. However, as the number of conflicting objectives increases, the performance of most MOEAs is badly deteriorated [1]. In case of Pareto-based MOEAs, these difficulties are intrinsically related to the fact that as the number of objectives increase, the proportion of non-dominated elements in the population grows, being increasingly difficult to discriminate among solutions using only the dominant relation [2].

Many-objective optimization evolutionary algorithms (MaEAs) refer to optimization problems greater than 4 [3]. Due to minimize and maximization problem can be mutual transformation, therefore, without loss of generality, this article mainly describes minimize multi-objective problem and its related concepts MaEAs can be defined as follows:

$$
\begin{gathered}
\text { Minimize } \mathrm{F}(\mathrm{x})=\left(\mathrm{f} 1(\mathrm{x}),\left(\mathrm{f} 2(\mathrm{x}) \ldots\left(\mathrm{f}_{\mathrm{m}}(\mathrm{x})\right) \mathrm{T}\right.\right. \\
\text { Subject to } x \in \Omega
\end{gathered}
$$

Where $\Omega \subset R^{n}$ is the feasible search region, $\mathrm{x}=(\mathrm{x} 1, \mathrm{x} 2 \ldots$ $\left.x_{n}\right) T$ is the decision variable vector, $f_{i}: R_{n}>R, i-1,2 \ldots m$ are the $\mathrm{m}$ objective functions, and $\mathrm{R}_{\mathrm{m}}$ is the objective space.

Classical optimization methods may fail to do so especially when the objective functions are nondifferentiable and without closed forms. For this reason, people resort to heuristic optimization methods such as evolutionary (EAs). Multi-objective evolutionary algorithms (MOEAs) have been attracting considerable attention. The number of MOEAs can be classified as three categories: (1) the decomposition-based approaches [4], [5] and [6]; (2) the indicator-based approaches
[7], [8] and [9]; and (3) the objective aggregation-based approaches [10], [11] and [12].

Biogeography/Complex based optimization(BBO/complex) algorithm is a kind of adaptive decomposition method. Detailed explanations of $\mathrm{BBO} /$ complex are introduced in Section II. However, the performance and convergence rate of $\mathrm{BBO} /$ complex is still to be further improved. With the migration flow of $\mathrm{n}$ SIV between rich and poor islands, we need a method to enhance its exploration and evaluate the badly modified whether to be accepted or not, it can prevent the past features always be overwritten by the newly emigrated features from other islands. On the other hand, since there are plenty of targets and constraints in the subsystem, when sharing information in the subsystem, we need a new method to reduce the computation time of the CPU. The simulated annealing (SA) algorithm was presented by Kirkpatrick et al. [13] and Valdo Cerny [14], SA algorithm is an intelligent algorithm that randomly search optimization based on probability. It is having the capacity of probabilistic jumping and it is able to accept non-inferior solutions and inferior solutions. Thus, effectively avoid falling into minimal local solutions. We are inspired here by Metropolis criterion of SA algorithm to solve the problem posed above. Details about SA will be introduced in next section.

\section{BBO FOR COMPLEX SYSTEMS AND SIMULATIED ANNEALING}

\section{A. BBO for Complex Systems}

BBO was invented less than a decade ago, but according to [15] to provide competitive optimization performance with ACO [16], differential evolution (DE) [17], particle swarm optimization (PSO) [18], and many other algorithms. Complex systems contain more than one subsystem, each of which is partially independent of the others. BBO/Complex is extending BBO to systems with multi-subsystems, where each subsystem contains multi-objectives and multi-constraints. The environment of $\mathrm{BBO} / \mathrm{Complex}$ includes $\mathrm{n}$ archipelagos, where $\mathrm{n}$ is the number of subsystems. Every archipelago consists of islands. The islands represent possible solutions to the problem. The structure of BBO/Complex is conceptually different from other typical algorithm. It includes both the framework and the optimization algorithm, as showed in Figure 1. It provides an efficient way to communicate between subsystems and provides a unique migration strategy to share information both within and across subsystems. 


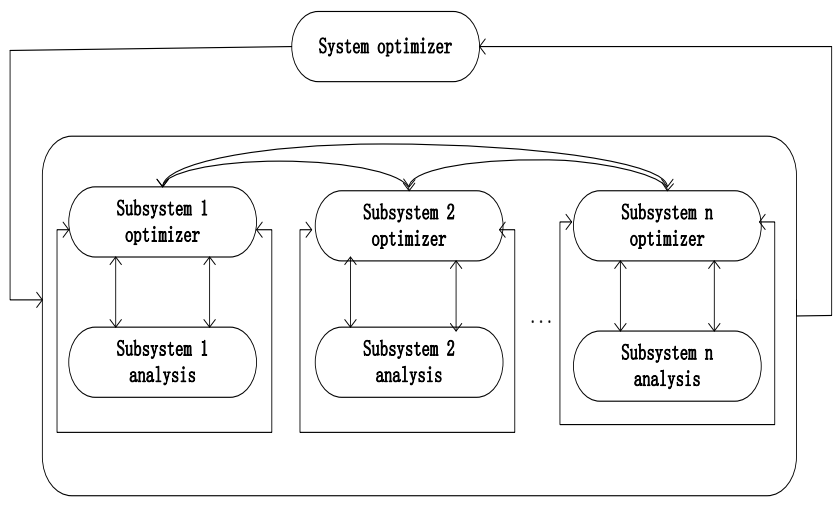

Fig 1: BBO/Complex formulation

\section{B. Migration algorithm}

Migration in $\mathrm{BBO} / \mathrm{Complex}$ needs to be modified due to the fact that the environment of $\mathrm{BBO} / \mathrm{Complex}$ contains more than one subsystem. Each subsystem contains multi-objectives and multi-constraints. The BBO/Complex's migration stage contains two types: with-subsystem and cross-subsystem. The islands with better part distance will have a better chance to be selected as the emigrating island. The migration processes are described by Algorithm 1.

\begin{tabular}{l} 
migration \\
\hline 1: Initialization $\lambda \mathrm{i}$ and $\mu \mathrm{i}$ for each member. \\
2: Perform with-subsystem migration: probabilistically choose the \\
Immigrating islands based on the islands ranks. \\
Use roulette wheel selection based on the emigration rates to select the \\
Emigrating island. \\
3: Perform cross-subsystem migration: find suitable pairs of subsystems \\
Based on similarity levels. Calculate distances between each pair of \\
Islands from different subsystems. Use roulette wheel selection \\
Based on partial distances to select the emigration islands.
\end{tabular}

\section{Mutation algorithm}

In BBO/Complex, there events are modeled as SIV mutation. The mutation rate $\mathrm{m}_{\mathrm{i}}$ can be determined by involving the species count probabilities $\mathrm{P}_{\mathrm{i}}$ into the following equation:

$$
m_{i}=m_{\max }\left(1-\frac{p i}{p \max }\right)
$$

Where the $\mathrm{P}_{\max }=\max$ (pi) and $\mathrm{m}_{\max }$ is a user-defined maximum mutation rate that mi can reach. The mutation is described by Algorithm 2.

\begin{tabular}{l} 
mutation \\
\hline 1:for $\mathrm{i} \leftarrow 1$ to $\mathrm{k}$ do \\
$\quad\{$ where $\mathrm{k}=$ number of islands or individuals $\}$ \\
2: $\quad$ Calculate probability Pi based on $\lambda_{\mathrm{i}}$ and $\mu_{\mathrm{i}}$ \\
$\quad$ \{by iterative or eigenvector method $\}$ \\
3: $\quad$ Calculate mutation rate mi \\
4: if rand $<$ mi and $\mathrm{i} \geq \mathrm{R}_{\mathrm{m}}$ then $\left\{\mathrm{R}_{\mathrm{m}}\right.$ is a user defined mutation range $\}$ \\
5: Replace $\mathrm{n}$ SIV vector of $\mathrm{ISI}_{\mathrm{i}}$ with a randomly generated $\mathrm{n}$ SIV vector \\
6: end if \\
7:end for
\end{tabular}

\section{Simulated annealing algorithm}

Algorithm SA is a meta-heuristic technique based on a thermodynamic process of the annealing of materials [22], [23], and [24]. The SA algorithm is constructed based on the statistical mechanics, which was demonstrated by Metropolis et al. in 1953[25] through the concept of Boltzmann's probability distribution. It means if a system is maintained in a thermal equilibrium at temperature $T$, then the probability distribution $p$ of its energy $E$ can be achieved by [26]:

$$
P(E)=e \frac{-\Delta E}{k_{B} T}
$$

Where KB is a Boltzmann's constant. The difference in energy $\Delta E$ means the difference in cost function between the past and current iterations, which can be determined as follows:

$\Delta E=f\left(x_{n}\right)-f\left(x_{o}\right)$

For minimization problems, $\Delta E \leqslant 0$ means $f\left(x_{n}\right) \leq f\left(x_{o}\right)$, so the new design point is directly accepted. Otherwise, the Metropolis criterion will be enabled to decide whether to accept or reject Xo. For this case where $\Delta E>0$, the acceptance is treated probabilistically according with the relation $^{P}=\frac{1}{1+e^{(\Delta E / \max (T))}}$. It can be viewed the influence of temperature in the acceptance process. For the highest magnitudes of $\mathrm{T}$, The acceptance probability to choose a worse state is likewise higher. This process will avoid trapping into local optima. As the temperature decrease, the SA algorithm accepts only states which minimize the FO cost. Therefore, the way that temperature decreases during the iteration of the algorithm is an important parameter, this parameter is named cooling schedule [26].

\section{THE HYBRID BBO/COMPLEX ALGORITHM}

The proposed hybrid $\mathrm{BBO} /$ complex ( $\mathrm{HBBO} /$ complex) is described by Algorithm 3. When the migration stage is completed, the features ( $\mathrm{n} \mathrm{SIV)}$ of the islands will not be directly overwritten with the new values that come from the probabilistically selected source inlands. Instead, there n SIV of the islands is saved in two temporary matrices. Each row of their matrices represents one individual. The old independent variables are used again if and only if the modified individual shows lower solution quality and does not meet the Metropolis criterion. With this restriction on the migration stage, the overall performance of the HBBO/Complex algorithm can be enhanced. By this method, the exploration of the $\mathrm{BBO} /$ complex algorithm is greatly improved.

\section{HBBO/Complex}

Initialization stage with all the parameters,

1: Decompose the complex systems based on the system requirements;

2: Compute the constraint violations of all islands;

3: Do migration

4: While $\left(\mathbf{T}>\mathbf{T}_{\text {ini }}\right)$

5: Calculate $\Delta E=\mathrm{V} 2(\mathrm{i})-\mathrm{V} 1(\mathrm{i})$

6: if $\Delta E>0$ then

7: Apply the Metropolis criterion

8: if $\mathrm{P}(\mathrm{E})>$ rand then

9: Re-select the past $(I, 1 \rightarrow n)$ vector of matrix as an updated population for ISI $_{i}$

10: end if

11: end if

12 : end for

13 Update the population with sorting and mapping.

14: Do mutation.

15: Do clear duplicated SIV

16: Replace the worst ISI with the good ISI saved in the elitism stage 


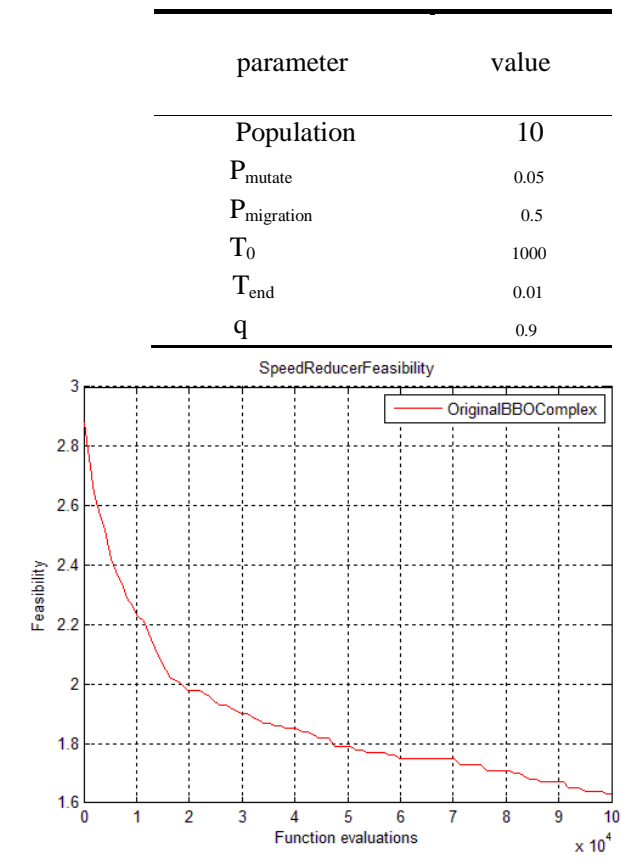

\section{SimUlation RESULTS}

In this section, we compare the performance of HBBO/Complex in real-world benchmark problems with Original BBO/Complex and Collaborative optimization (CO) The benchmark problems are obtained from [22] and include the speed reducer problem. It is contains several subsystems and multi-constraints. The speed reducer problem is a gear box design problem. The objective is to minimize the gear box weight and the von Mises stresses for shafts 1 and 2. It contains 3 objectives, 11 constraints, and 7 design variables. This problem is defined as follows:

Min $\mathrm{F} 1=0.7854 \mathrm{X}_{1} \mathrm{X}_{2}^{2}\left(3.3333 \mathrm{X}_{3}^{2}+14.9334 \mathrm{X}_{3}-43.0934\right)-$ $1.05079 \mathrm{X}_{1}\left(\mathrm{X}_{6}{ }^{2}+\mathrm{X}_{7}^{2}\right)+7.477\left(\mathrm{X}_{6}^{3}+\mathrm{X}_{7}^{3}\right)+0.7845\left(\mathrm{X}_{4} \mathrm{X}_{6}^{2}+\mathrm{X}_{5} \mathrm{X}_{7}^{2}\right)$,

$$
\begin{aligned}
& \text { MinF } 2=\sqrt{\left(\frac{745 x_{4}}{x_{2} x_{3}}\right)^{2}+1.69 \times 10^{7}}, \\
& \text { MinF3 }=\sqrt{\left(\frac{745 x_{5}}{x_{2} x_{3}}\right)^{2}+1.575 \times 10^{8}},
\end{aligned}
$$

Such that the following constraints hold:

$$
\begin{aligned}
& \mathrm{g}_{1=}, \frac{27}{x_{1} x_{2}{ }^{2} x_{3}}-1 \leq \mathrm{O} \\
& \mathrm{g}_{2}=\frac{397.5}{x_{1} x_{2}{ }^{2} x_{3}}-1 \leq \mathrm{O} \\
& \mathrm{g}_{3}=\frac{1.93 x_{4}{ }^{3}}{x_{2} x_{3} x_{6}{ }^{4}}-1 \leq \mathrm{O} \\
& \mathrm{g}_{4}=\frac{1.93 x_{5}{ }^{3}}{x_{2} x_{3} x_{7}{ }^{4}}-1 \leq \mathrm{O} \\
& \mathrm{g}_{5}=\sqrt{\frac{\left(745 x_{4} / x_{2} x_{3}\right)+1.69 \times 10^{7}}{0.1 x_{6}{ }^{3}}}-1100 \leq 0 \text {, } \\
& g_{6}=\sqrt{\frac{\left(745 x_{5} / x_{2} x_{3}\right)+1.575 \times 10^{8}}{0.1 x_{6}^{3}}}-850 \leq 0 \text {. } \\
& \mathrm{g}_{7}=x_{2} x_{3}-40 \leq 0 \\
& \mathrm{~g}_{8}=\frac{x_{1}}{x_{2}}-12 \leq \mathrm{O} \text {, } \\
& \mathrm{g}_{9}=\frac{-x_{1}}{x_{2}}+4 \leq 0 \text {, } \\
& \mathrm{g}_{10}=\frac{1.5 x_{6}+1.9}{x_{4}}-1 \leq 0 \\
& \mathrm{~g}_{11}=\frac{1.1 x_{7}+1.9}{x_{5}}-1 \leq 0
\end{aligned}
$$
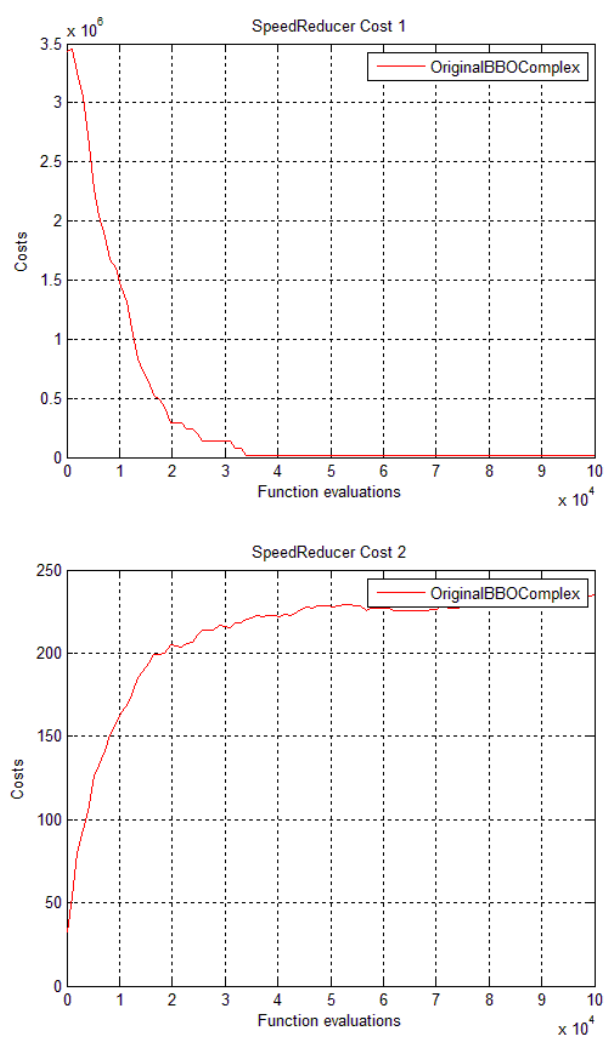

Table 1 show the parameters used in the HBBO/Complex.

Table 1: Simulation parameters of the HBBO/Complex algorithms 


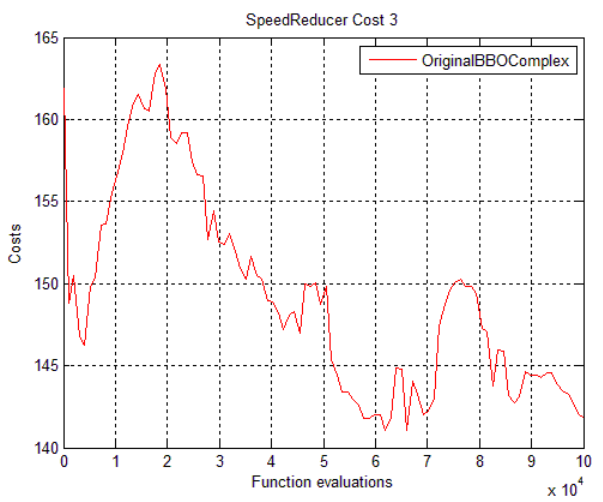

Fig 2: The original BBO/Complex algorithm feasibility and cost of each objective for the speed reducer problem
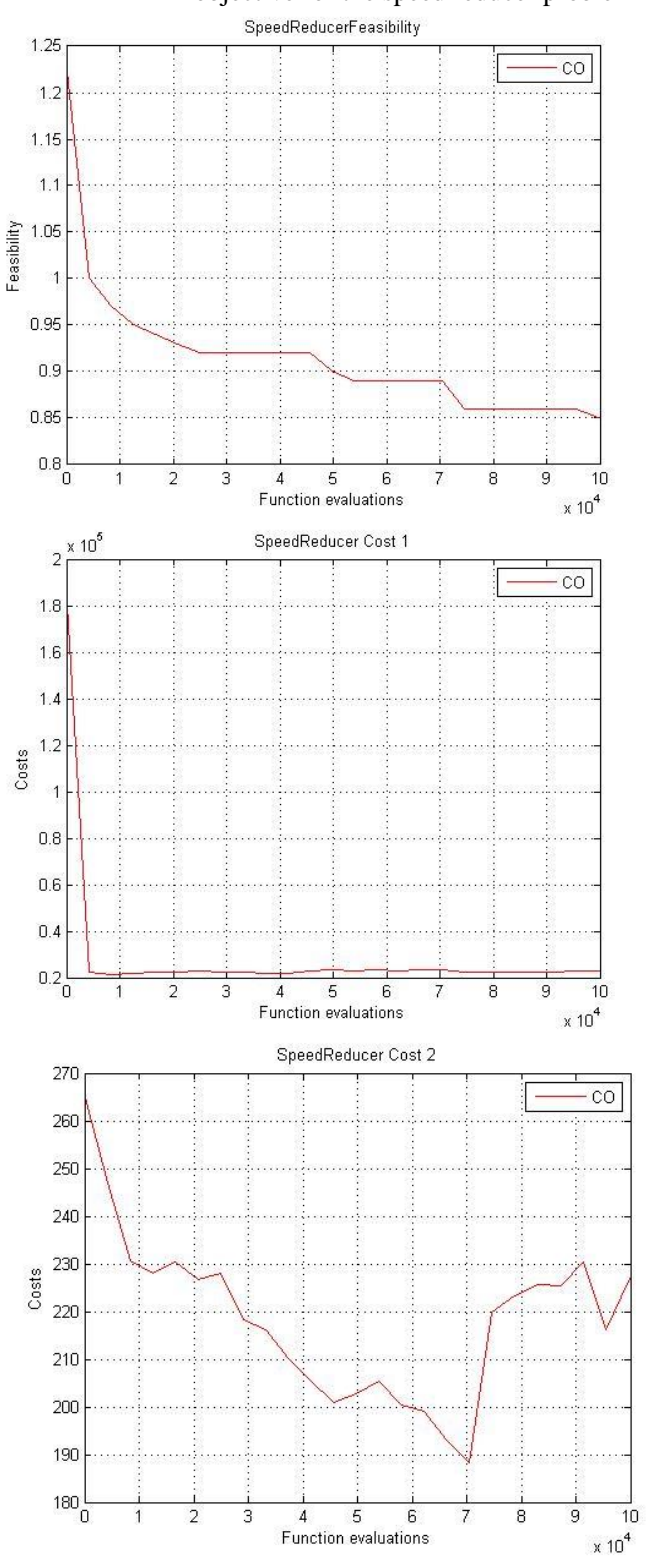

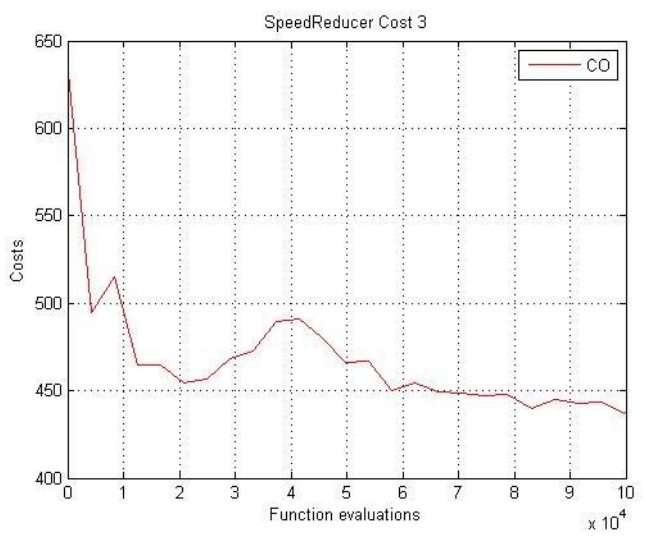

Fig 3: The CO algorithm feasibility and cost of each objective for the speed reducer problem

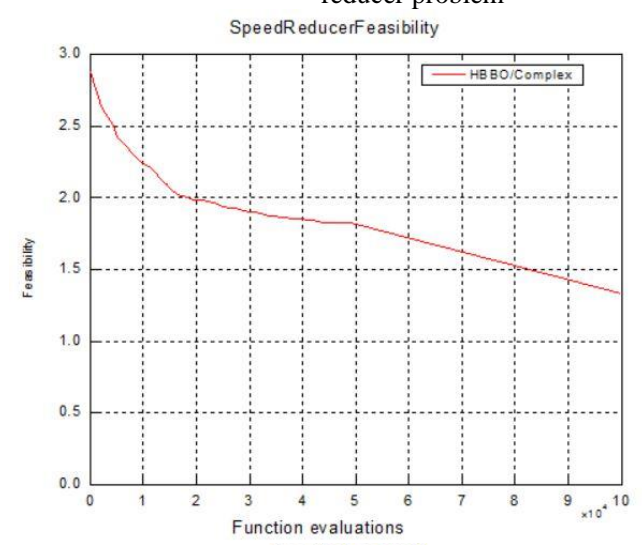

SpeedReducer cost
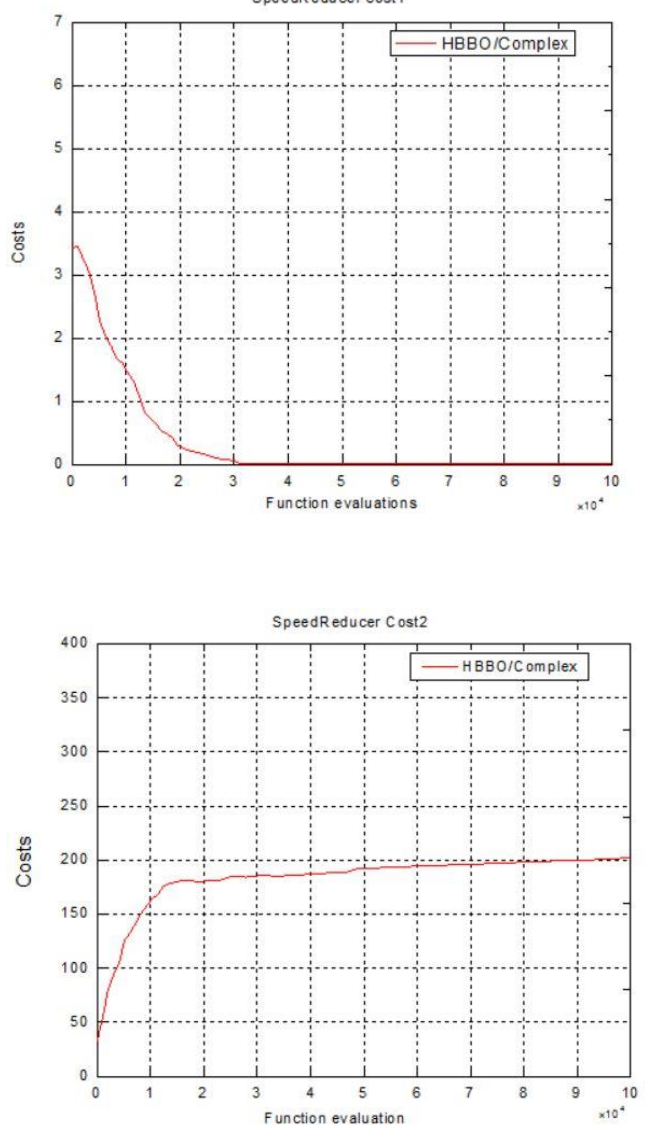


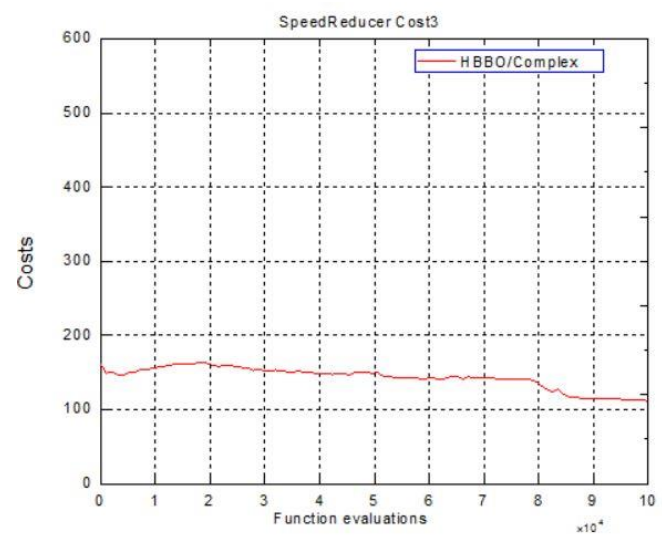

Fig 4: The HBBO/Complex algorithm feasibility and cost of each objective for the speed reducer problem

\section{CONCLUSIONS}

In this paper, we propose a novel complex system solution called $\mathrm{HBBO} / \mathrm{Complex}$. We compare the HBBO/Complex, $\mathrm{CO}$ and original BBO/Complex algorithm. The figures also show that the performance of $\mathrm{HBBO} / \mathrm{Complex}$ is superior to other two algorithms. With this process, the old features will not always be overwritten by the newly emigrated features from other islands. Instead, the Metropolis criterion is used to evaluate the badly modified populations whether they can be accepted or not. It has more flexible decomposition optimization options compared to $\mathrm{CO}$ and original $\mathrm{BBO} /$ Complex algorithm.

The obtained results show the performance of HBBO/Complex is markedly affected by introduced the SA algorithm. In general, aiming at many subsystems, manyobjectives and many-constraints problems for complex systems this hybrid algorithm raises the algorithm's immunity level against trapping into local optima.

\section{ACKNOWLEDGMENT}

This work was supported by the Hubei Provincial Department of Education Key Foundation, China(No.D20151803), the Hubei Provincial Department of Education Instructive Foundation ,China(No B2016084).

\section{REFERENCES}

[1] Eckart Zitzler, Marco Laumanns, Lothar Thiele. SPEA2: Improving the Strength Pareto Evolutionary Algorithm for Multiobjective Optimization[C]// Evolutionary Methods for Design, Optimization and Control with Applications To Industrial Problems. Proceedings of the Eurogen'2001. Athens. Greece, September. 2001.

[2] Ishibuchi H, Tsukamoto N, Nojima Y. Evolutionary many-objective optimization: A short review $[\mathrm{C}] / /$ Evolutionary Computation. 2008:2419-2426.

[3] Lücken C V, Barán B, Brizuela C. A survey on multi-objective evolutionary algorithms for many-objective problems[J]. Computational Optimization \& Applications, 2014, 58(3):707-756.

[4] Zhang Q, Li H. MOEA/D: A Multiobjective Evolutionary Algorithm Based on Decomposition[J]. IEEE Transactions on Evolutionary Computation, 2008, 11(6):712-731.
[5] Hughes E J. MSOPS-II: A general-purpose Many-Objective optimiser[C]// Evolutionary Computation, 2007. CEC 2007. IEEE Congress on. 2007:3944-3951.

[6] Hughes E J. Multiple single objective Pareto sampling[C]// Evolutionary Computation, 2003. CEC '03. The 2003 Congress on. 2004:2678-2684 Vol.4.

[7] Beume N, Naujoks B, Emmerich M. SMS-EMOA: Multiobjective selection based on dominated hypervolume $[\mathrm{J}]$. European Journal of Operational Research, 2007, 181(3):1653-1669.

[8] Bader J, Zitzler E. HypE: An Algorithm for Fast Hypervolume-Based Many-Objective Optimization[J]. Evolutionary Computation, 2011, 19(1):45-76.

[9] Zitzler E, Künzli S. Indicator-Based Selection in Multiobjective Search[J]. Lecture Notes in Computer Science, 2015, 3242:832-842.

[10] Kruisselbrink J W, Emmerich M T M, Bäck T, et al. Combining Aggregation with Pareto Optimization: A Case Study in Evolutionary Molecular Design[C]// Evolutionary Multi-Criterion Optimization, International Conference, Emo 2009, Nantes, France, April 7-10, 2009. Proceedings. 2009:453-467.

[11] Zitzler E, Thiele L. Multiobjective evolutionary algorithms: a comparative case study and the strength Pareto approach[J]. IEEE Transactions on Evolutionary Computation, 1999, 3(4):257-271.

[12] Murata T, Taki A. Many-Objective Optimization for Knapsack Problems Using Correlation-Based Weighted Sum Approach[C]// Evolutionary Multi-Criterion Optimization, International Conference, Emo 2009, Nantes, France, April 7-10, 2009. Proceedings. 2009:468480 .

[13] Kirkpatrick S, Gelatt C D, Vecchi M P. Optimization by Simulated Annealing[J]. Science, 1983, 220(4598):671-80.

[14] V. Černý. Thermodynamical approach to the traveling salesman problem: An efficient simulation algorithm[J]. Journal of Optimization Theory \& Applications, 1985, 45(1):41-51.

[15] Simon D. Biogeography-Based Optimization[J]. IEEE Transactions on Evolutionary Computation, 2009, 12(6):702-713.

[16] Zhang Z, Gao C, Lu Y, et al. Multi-Objective Ant Colony Optimization Based on the Physarum-Inspired Mathematical Model for Bi-Objective Traveling Salesman Problems.[J]. Plos One, 2016, 11(1).

[17] Guo X, Wang Y, Wang X. An objective reduction algorithm using representative Pareto solution search for many-objective optimization problems[J]. Soft Computing, 2015:1-15.

[18] Sun X, Xu R, Zhang Y, et al. Sets evolution-based particle swarm optimization for many-objective problems[C]// IEEE International Conference on Information and Automation. IEEE, 2014:1119-1124.

[19] Du D, Simon D. Complex System Optimization Using BiogeographyBased Optimization[J]. Mathematical Problems in Engineering, 2013 2013(1-2):1-17.

[20] Guo W, Wang L, Wu Q. An analysis of the migration rates for biogeography-based optimization[J]. Information Sciences, 2014, 254(19):111-140.

[21] Hathaway R J, Bezdek J C. Fuzzy c-means clustering of incomplete data.[J]. IEEE Transactions on Systems Man \& Cybernetics Part B Cybernetics A Publication of the IEEE Systems Man \& Cybernetics Society, 2001, 31(5):735-744.

[22] Kirkpatrick S, Gelatt C D, Vecchi M P. Optimization by Simulated Annealing[J]. Science, 1983, 220(4598):671-80.

[23] Metropolis N, Rosenbluth A W, Rosenbluth M N, et al. Equation of State Calculations by Fast Computing Machines[J]. Journal of Chemical Physics, 2004, 21(6):1087-1092.

[24] Eglese R W. Simulated annealing: A tool for operational research[J]. European Journal of Operational Research, 1990, 46(3):271-281.

[25] Senga S. Engineering Optimization Theory and Practice[J]. History of Economic Thought, 1996, 52(5):27-45.

[26] Gonzales G V, Estrada E D S D, Emmendorfer L R, et al. A comparison of simulated annealing schedules for constructal design of complex cavities intruded into conductive walls with internal heat generation[J]. Energy, 2015, 93(P1):372-382. 